\title{
Energy Harvesting for Self-Powered Nanosystems
}

\author{
Zhong Lin Wang $(\bowtie)$ \\ School of Materials Science and Engineering, Georgia Institute of Technology, Atlanta, GA 30332-0245, USA \\ Received: 24 April 2008/ Revised: 12 May 2008/Accepted: 12 May 2008 \\ CTsinghua Press and Springer-Verlag 2008
}

\begin{abstract}
In this article, an introduction is presented about the energy harvesting technologies that have potential for powering nanosystems. Our discussion mainly focuses on the approaches other than the well-known solar cell and thermoelectrics. We mainly introduce the piezoelectric nanogenerators developed using aligned $\mathrm{ZnO}$ nanowire arrays. This is a potential technology for converting mechanical movement energy (such as body movement, muscle stretching, blood pressure), vibration energy (such as acoustic/ultrasonic wave), and hydraulic energy (such as flow of body fluid, blood flow, contraction of blood vessel, dynamic fluid in nature) into electric energy for self-powered nanosystems.
\end{abstract}

\section{KEYWORDS}

Nanogenerator, self-powered nanosystem, energy harvesting

\section{Introduction}

Exploring renewable, sustainable and green energy resources is the most critical challenge to sustainable development of human civilization [1,2]. At the large scale, besides the well-known energy resources that power the world today, such as petroleum, coal, hydraulic, natural gas and nuclear, active research and development are being taken in exploring alternative energy resources such as solar, geothermal, biomass, nuclear, wind, and hydrogen. At a much smaller scale, energy and technologies are desperately needed for independent and continuous operations of implantable biosensors, ultrasensitive chemical and biomolecular sensors, nanorobotics, microelectromechanical systems, remote and mobile environmental sensors, homeland security and even portable personal electronics. A nanorobot, for example, is proposed to be a smart machine that may be able to sense and adapt to the environment, manipulate objects, taking actions, and perform complex functions, but a key challenge is to find a power source that can drive the nanorobot without adding much weight. An implanted wireless biosensor, for example, requires a power source, which may be provided directly or indirectly by charging of a battery. It is highly desired for wireless devices and even required for implanted biomedical devices to be self-powered without using battery. Therefore, it is desperate to develop nanotechnology that harvests energy from the environment for selfpowering these nanodevices. This is a key step towards self-powered nanosystems [3].

A key advantage of nanodevices and nanosystems is that they usually operate at a very low power in the range from $\mathrm{nW}$ to $\mu \mathrm{W}$. As a result, the energy harvested from the environment may be sufficient to power the system. Figure 1 shows a biochemical

Address correspondence to zlwang@gatech.edu

\section{算 Springer}




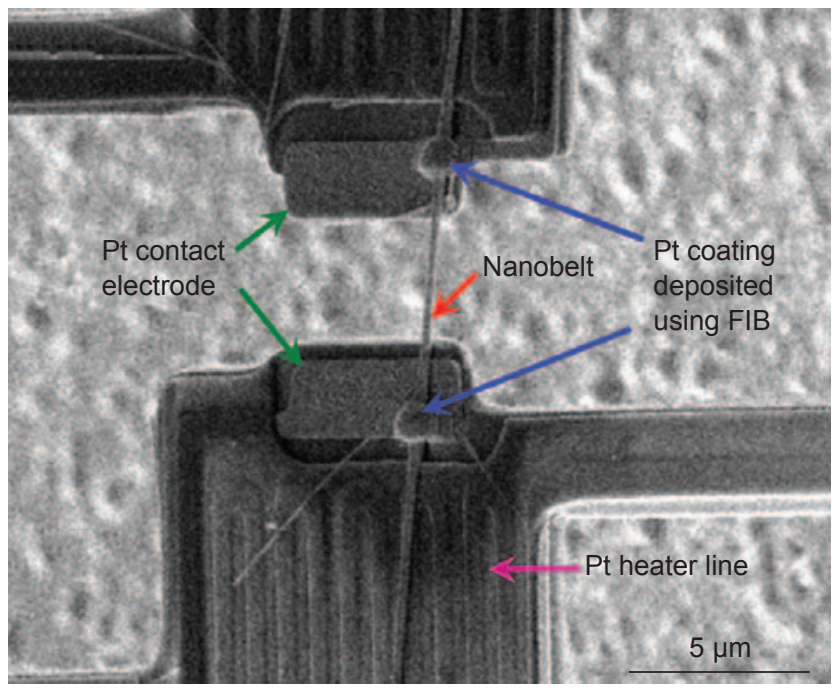

(a)

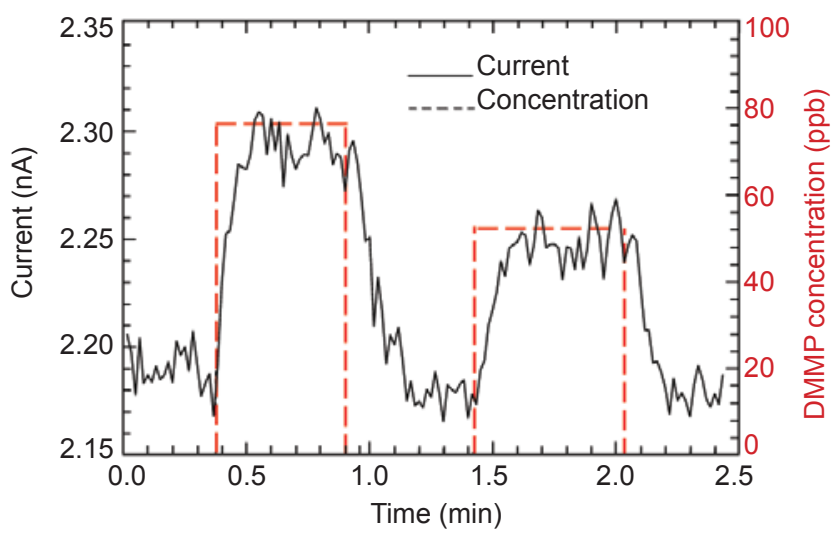

(b)

Figure 1 (a) Scanning electron microscopy (SEM) image of a single tin oxide nanobelt fabricated chemical sensor. (b) Response of the as-assembled nanobelt sensor to 78 and 53 ppb DMMP. The voltage applied to the nanobelt was $1.5 \mathrm{~V}$ (data courtesy Prof. Li Shi)

sensor built used a single $\mathrm{SnO}_{2}$ nanobelt for detecting dimethyl methylphosphonate (DMMP), a nerve agent stimulant, at $50 \mathrm{ppb}$ level [4]. The sensor operates at $1.5 \mathrm{~V}$ and the electrical current is in the range of 2$3 \mathrm{nA}$, which means the power needed to operate this nanosensor is $5 \mathrm{nW}$ excluding the heating unit.

Scientists have developed various approaches for scavenging energy for mobile and wireless microelectronics using thermoelectrics, mechanical vibration, and piezoelectric (PZ) vibration $[5,6]$. Photovoltaic is one of the most well-established energy scavenging technology, which converts solar energy into electricity with the use of a photonelectron excitation process in semiconductor materials. A human body provides numerous potential power sources-mechanical energy, vibration energy, chemical energy (glucose), and hydraulic energy. If a small fraction of such energy could be converted into electricity, the energy may be sufficient to power small devices for biomedical devices [7]. We now review a few approaches that have been developed for energy harvesting at small scales.

\section{Microbial fuel cells}

Micro-organisms can be used to transform bioconvertible substrates directly into electricity, in which the bacterium acts as an anode and the electrons flow from the cathode through a resistor [8]. The catalytic actions of micro-organisms have been used to produce electrical output from the different carbohydrates and complex substrates. The implantable direct glucose fuel cell can produce power output in the range of $50 \mu \mathrm{W}$, sufficient to supply a cardiac pacemaker. Animal trials of direct glucose fuel cells have shown durability of 150 days. Both glucose and oxygen are present in the cell and tissue of all eukaryotic organisms, including human beings. Therefore, it is possible to tap into the body's own resources, including the metabolic properties of our cells, to generate enough energy to power an array of clinical devices, including drug delivery systems, diagnostic tools, and human augmentation devices.

\section{Adenosine tri-phosphate energy converter}

Enzymes are proposed as an alternative to powering future nanomechanical devices. Possible nanoscale biological motor enzymes that could be used are kinesin [9], RNA polymerase [10], myosin [11], and adenosine tri-phosphate (ATP) syntase [12,13]. The motors are fueled by ATP molecule, which consists of adenine, ribose, and three phosphate groups that are linked by covalent bonds. As the first phosphate group is removed, which is signaled by a coenzyme, a large amount of energy is released with the form of a reaction product called adenosine diphosphate (ADP). If a further amount of energy is desirable, the second phosphate group is released to create adenosine monophosphate (AMP). The 
energy created is made available to be used for chemosynthesis, locomotion (including muscle contraction in animals), and the active transport of ions and molecules across cell membranes. ATP is refueled by the rephosphorylation of ADP and AMP using the chemical energy generated from the oxidation of food. This concept leads to ATP serving as rechargeable batteries inside the human body.

\section{Thermoelectric generator}

Thermoelectric generator relies on the Seebeck Effect, which is about the electric potential observed flowing between two dissimilar metals that form a junction and are at different temperatures. The voltage produced is proportional to the temperature difference between the two ends. The proportionality constant is known as the Seebeck coefficient, and often referred to as the thermoelectric power or thermopower. This is the physical basis for a thermocouple, which is used often for temperature measurement. Seebeck coefficient of a material characterizes the magnitude of an induced thermoelectric voltage owing to a temperature difference present across the material. An applied temperature difference causes charged carriers in the material, whether they are electrons or holes, to diffuse from the hot side to the cold side, similar to a classical gas that expands when heated. Mobile charged carriers migrating to the cold side leave behind their oppositely charged and immobile nuclei at the hot side thus giving rise to a thermoelectric voltage.

Thermoelectrics is one of the most exciting fields in nanotechnology [14]. One dimensional nanomaterials that have a small thermal conductivity but high electrical conductivity, such as Bi and BiTe, are very beneficial for improving the thermal power. Thermoelectric generator usually has a large size because of the requirements of maintaining a highertemperature difference between the two ends of the device.

\section{Vibration-based energy generation}

Scientists have built vibration-based generators using three types of electromechanical transducers:

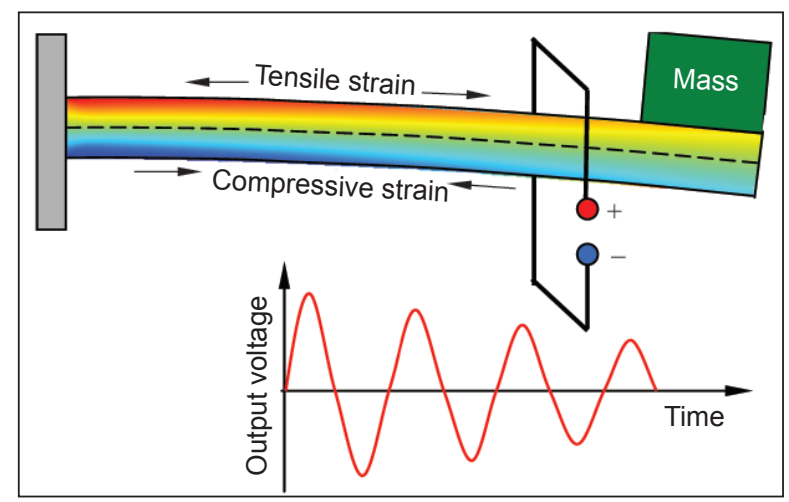

Figure 2 Schematic diagram showing a piezoelectric vibrationbased generator for producing $A C$ voltage output

electromagnetic, electrostatic, and piezoelectric. The electromagnetic microgenerator utilizes a moving magnet or coil for inducting and alternating electric current in a close circuit. Although some microgenerators have been fabricated at the scale of micro-electromechanical systems (MEMS), the technology tends to produce large structures ranging of $1-75 \mathrm{~cm}^{3}$, exploring vibration ranges from $50 \mathrm{~Hz}$ to $5 \mathrm{kHz}$ that induce mechanical oscillations between one-half micrometer and over one millimeter, and producing power from tens of $\mu \mathrm{W}$ to over a $\mathrm{kW}$.

A typical piezoelectric vibration-based generator uses a double layered piezoelectric beam with a mass at the end (Fig. 2). The principle of this design is based on piezoelectricity, which is the ability of certain crystals to generate a voltage in response to applied mechanical stress. When the gravitation attracts the beam to bend downward, the upper piezoelectric layer is under tensile strain and the lower layer is under compressive strain, resulting in a positive and negative voltage, respectively, across the beam. The mass oscillates back and forth; an alternating voltage would be output. This approach has been the basic principle for converting mechanical vibration energy into electricity for microsystems. This energy generator is reasonably large so that gravitation plays a major role for drive the oscillation of the mass. This design has been one of the major microgenerators for mobile and wireless electronics.

\section{Piezoelectric nanogenerators}

As shown in Fig. 2, if the size of the beam is in 
the nanometer scale, gravitation plays almost no role in driving the motion of the mass, so that the proposed generator would not work. For nanoscale energy source, innovations are needed for delivering nanoscale power source so that the nanodevices and nanosystems can work independently. We have explored innovative nanotechnologies for converting mechanical energy (such as body movement, muscle stretching), vibration energy (such as acoustic/ ultrasonic wave), and hydraulic energy (such as body fluid and blood flow) into electric energy that will be used to power nanodevices without using battery [15]. Our study is based on aligned $\mathrm{ZnO}$ nanowires (NWs) grown on a conductive solid substrate (Fig. 3(a)). The measurements were performed by an atomic force microscope (AFM) using a Si tip coated with Pt film. In the AFM contact mode, a constant normal force of $5 \mathrm{nN}$ was maintained between the tip and sample surface (Fig. 3(b)). The tip scanned over the top of the $\mathrm{ZnO} \mathrm{NW}$, and the tip's height was adjusted according to the surface morphology and local contacting force. In the corresponding voltage output image for each contact position, many sharp output peaks were observed (Fig. 3(c)).

The physical principle for creating, separating, and preserving the piezoelectric charges in the NW is

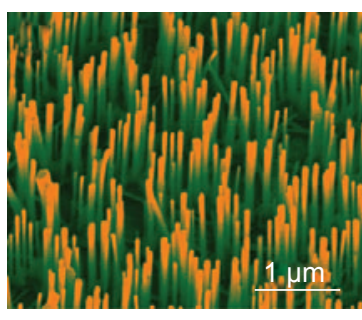

(a)

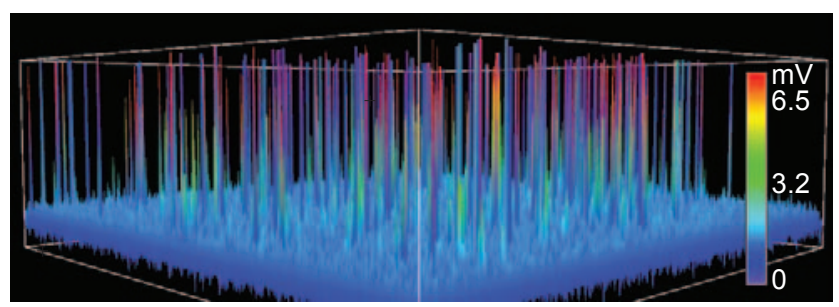

(c)

Figure 3 (a) Scanning electron microscopy (SEM) images of aligned ZnO NWs grown on sapphire substrate. (b) Experimental set up and procedures for generating electricity by deforming a piezoelectric NW using a conductive AFM tip. The AFM scans across the NW arrays in contact mode. (c) Output voltage image of the NW arrays when the AFM tip scans across the NW arrays a coupling of piezoelectric and semiconducting properties [16]. For a vertical, straight ZnO NW (Fig. 4(a)), the deflection of the NW by AFM tip creates a strain field, with the outer surface being tensile (positive strain $\varepsilon$ ) and inner surface compressive (negative strain $\varepsilon$ ) (Fig. 4(b)). A piezoelectric field $E_{z}$ along the NW is then created inside the NW volume by the piezoelectric effect, with the piezoelectric field is closely parallel to the NW at the outer surface and anti-parallel to the NW at the inner surface (Fig. 4(c)). As a result, across the width of the NW at the top end, the electric potential distribution from the compressed to the stretched side surface is approximately between $V_{\mathrm{s}}^{-}$(negative) to $V_{\mathrm{s}}^{+}$(positive).

We now consider the voltage output process. In the first step, the AFM conductive tip that induces the deformation is in contact with the tensile surface of positive potential $V_{\mathrm{s}}^{+}$(Figs. $4(\mathrm{~d})$ and (e)). The Pt metal tip has a potential of nearly zero, $V_{\mathrm{m}}=0$, so the metal tip- $\mathrm{ZnO}$ interface is negatively biased for $\Delta V=V_{\mathrm{m}}-$ $V_{\mathrm{s}}^{+}<0$. With consideration the n-type semiconductor characteristic of the as-synthesized $\mathrm{ZnO}$ NWs, the $\mathrm{Pt}$ metal-ZnO semiconductor (M-S) interface in this case is a reverse-biased Schottky diode (Fig. 4(e)) [A Schottky contact is a metal-semiconductor contact at which a potential barrier is formed, so that it behaves like a diode that only allows the current to flow from metal to semiconductor], and little current flows across the interface. This is the process of creating, separating and accumulating the charges. In the second step, when the AFM tip is in contact with the compressed side of the NW (Fig. 4(f)), the metal tip- $\mathrm{ZnO}$ interface is positively biased for $\Delta V=V_{\mathrm{m}}-V_{\mathrm{s}}^{-}>0$. The M-S interface in this case is a positively biased Schottky diode, and it produces a sudden increase in the output electric current. The current is the result of $\Delta V$ driven flow of electrons from the semiconductor $\mathrm{ZnO} N W$ to the metal tip. This is the charge releasing process.

The mechanism demonstrated in Fig. 4 is the fundamental principle of piezoelectric nanogenerators using nanowires, which have a few advantages. First, the NW-based nanogenerators can be subjected to extremely large deformation, so that it can be used for flexible electronics as flexible/foldable power source. Second, the large degree of deformation that can be 
supported by the NWs is likely to

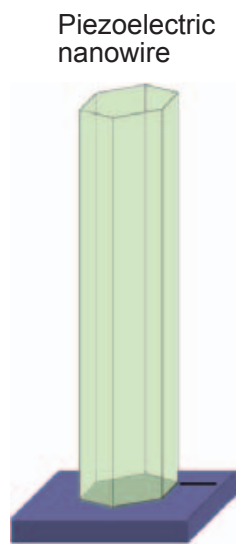

(a)

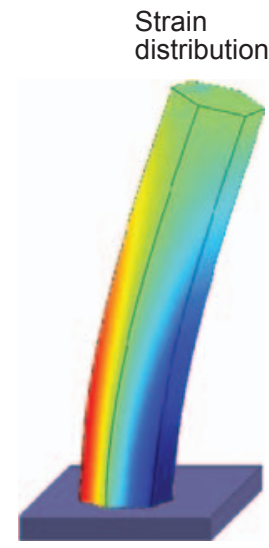

(b)

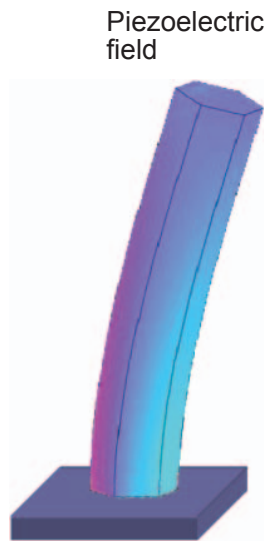

(c)

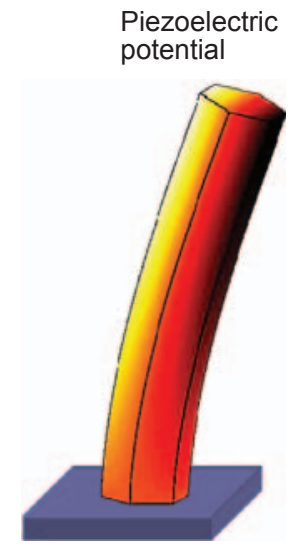

(d) energy (body movement, muscle stretching) in human body to generate electricity. Finally, $\mathrm{ZnO}$ is an environmental green material.

\section{Direct-current nanogen- erator driven by ultrasonic wave}

Although the approach presented in Fig. 3 has explored the principle and potential of the nanogenerator, for technological applications, we must make an innovative design to drastically improve the performance of the nanogenerator in following aspects. Firstly, we must eliminate the use of AFM for making the mechanical deformation of the NWs so that the power generation can be achieved by an adaptable, mobile and cost-effective approach over a larger scale. Secondly, all of the NWs are required to generate electricity simultaneously and continuously, and all the electricity can be effectively collected and output. Finally, the energy to be converted into electricity has to be provided in a form of wave/vibration from the environment, so the nanogenerator can operate "independently" and wirelessly. We have developed an innovative approach that has addressed these challenges [18].

The experimental set up is schematically shown in Fig. 5(a), in which an array of aligned ZnO NWs is covered by a zigzag Si electrode coated with

Pt. The Pt coating is not only for enhancing the conductivity of the electrode but also for creating a Schottky contact at the interface with $\mathrm{ZnO}$. The top electrode is composed of parallel zigzag trenches fabricated on a (001) orientated Si wafer and coated with a thin layer of $\mathrm{Pt}$ film. Then, the electrode was placed and manipulated above the NW arrays at a controlled distance, followed by a careful packaging. The top electrode is designed to achieve the coupling process and to replace the role played by the AFM tip as shown in Fig. 4(b), and its zigzag trenches are to act as an array of aligned AFM tips. 


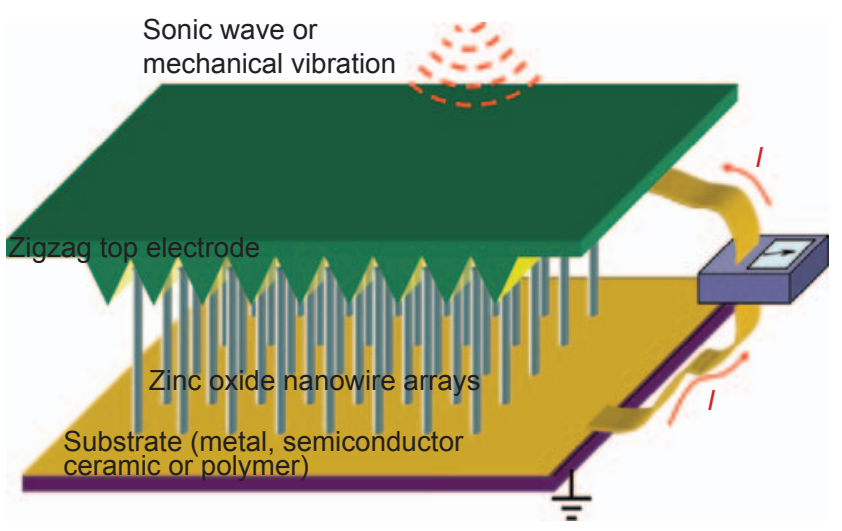

(a)

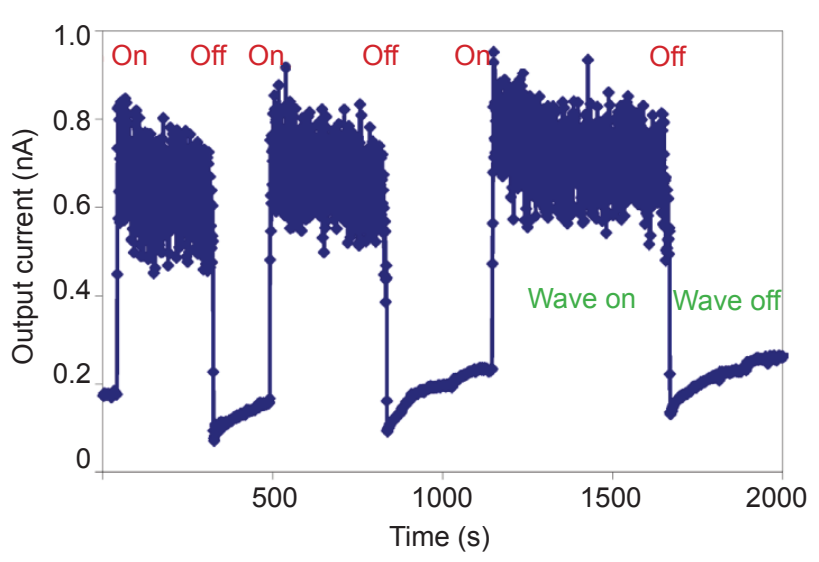

(b)

Figure 5 (a) Schematic diagram showing the direct current nanogenerator built using aligned ZnO nanowire arrays with a zigzag top electrode. The nanogenerator is driven by an external ultrasonic wave or mechanical vibration and the output current is continuous. (b) The output from a nanogenerator when the ultrasonic wave was turned on and off. The nanowires that simultaneously contributed to the DC current was estimated to be $\sim 500$

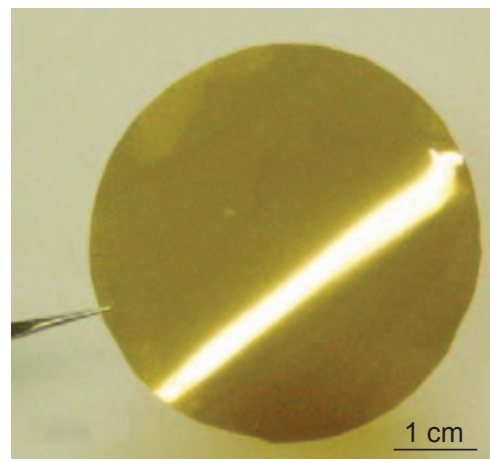

(a)

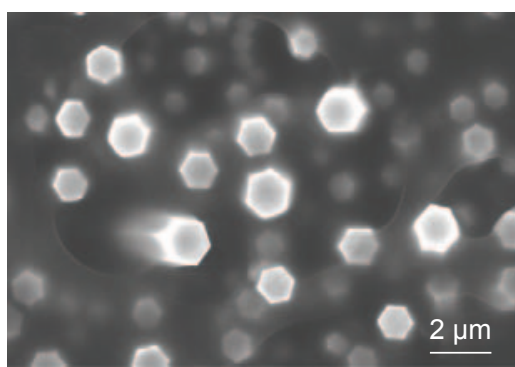

(c)

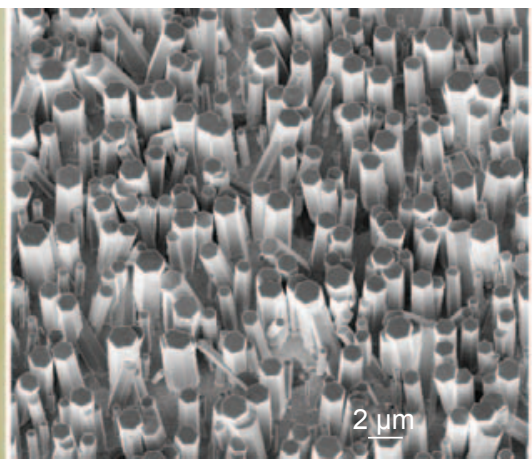

(b)

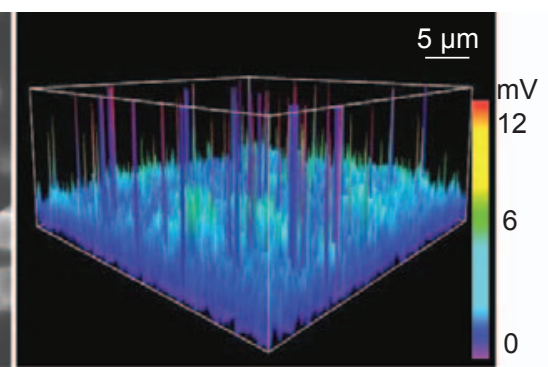

(d)

Figure 6 (a) Optical image of the polymer substrate on which aligned $\mathrm{ZnO}$ nanowires had been grown. (b) SEM image of the as-synthesized $\mathrm{ZnO}$ nanowires by a chemical approach. (c) SEM image of the as-synthesized ZnO nanowires on a patterned substrate. (d) Electric energy can be generated using the designed experiment demonstrated in Fig. 3, and electric output has been achieved

The electricity produced by the relative deflection/ displacement between the NWs and the electrode via either bending or vibration is expected to be output simultaneously and continuously. Figure 5(b) is an electric current output produced by $\sim 500 \mathrm{NWs}$ under the driving of ultrasonic wave. The current is on when the wave is on and is off when the wave is

off. This is the first demonstration of continuous current output produced by nanogenerator. The output of the DC nanogenerator has recently been improved to $500 \mathrm{nA}$ and $10 \mathrm{mV}$ [19].

\section{Flexible power generator built on polymer substrate}

The ceramic or semiconductor substrates used for growing $\mathrm{ZnO} \mathrm{NWs}$ are hard, brittle and cannot be used in the areas that require a foldable or flexible power source, such as implantable biosensors in muscles or joints, and power generator built in walking shoes [20]. It is necessary to use conductive polymer/plastics as substrate that is likely to be biocompatible and biosafe. Two advantages may be offered by this approach. One is the cost-effective, large-scale solution approach used to grow $\mathrm{ZnO} \mathrm{NW}$ arrays at a temperature lower than $80{ }^{\circ} \mathrm{C}$. The other is the large-degree of choice of flexible plastic substrates used for growing aligned $\mathrm{ZnO} \mathrm{NW}$ arrays, which could play an important role in the flexible and portable electronics. Highly aligned ZnO NWs have been grown using chemical synthesis. Figure 6(a) shows optical images of a large polymer substrate on which $\mathrm{ZnO}$ NW arrays were grown, which are 
clearly revealed by scanning electron microscopy image (Fig. 6(b)). To improve the adhesion of the substrate with the NWs, a thin layer of polymer can be spanned onto the substrate after the growth, so that the roots of the NWs are partially embedded (Fig. 6(c)). Electric energy can be generated using the designed experiment demonstrated in Fig. 3, and electric output of $\sim 10-40$ $\mathrm{mV}$ has been achieved (Fig. 6(d)). The piezoelectric power generators using $\mathrm{ZnO}$ NWs arrays on flexible plastic substrate might be able to harvest energy from the environment such as body movement (e.g., gestures, respiration, or locomotion).

\section{Fiber-based nanogenerators for power shirt}

To capture the energy that is available from biological systems, we need to develop nanogeneratgors that are built on flexible and foldable substrates and target at low frequency excitations (typically $\sim 10 \mathrm{~Hz}$ ) [21]. The design of the nanogenreator is based on the mechanism of utilizing zigzag electrode, but we have replaced the zigzag electrode by an array of metal wires, as shown

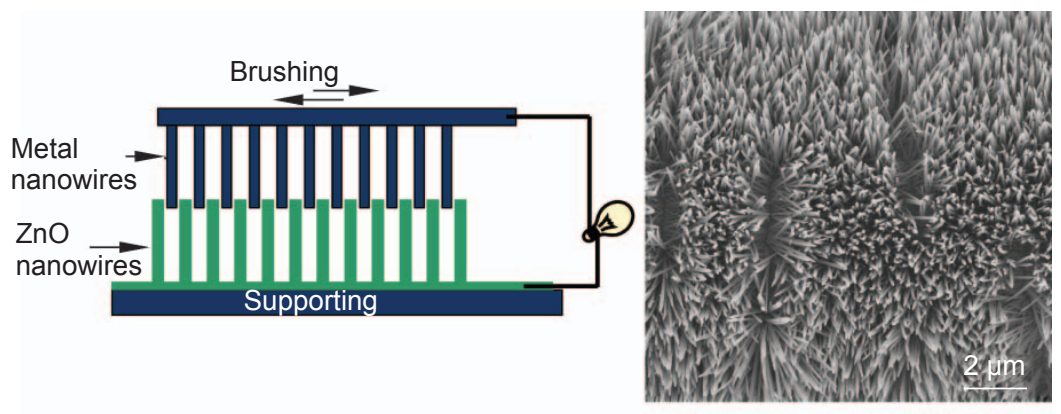

(a)

(b)

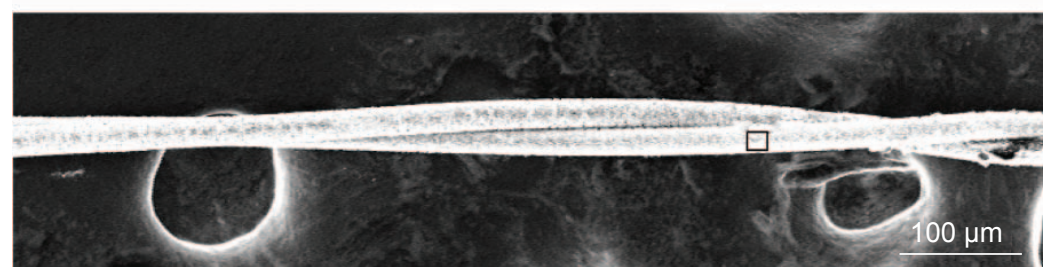

(c)

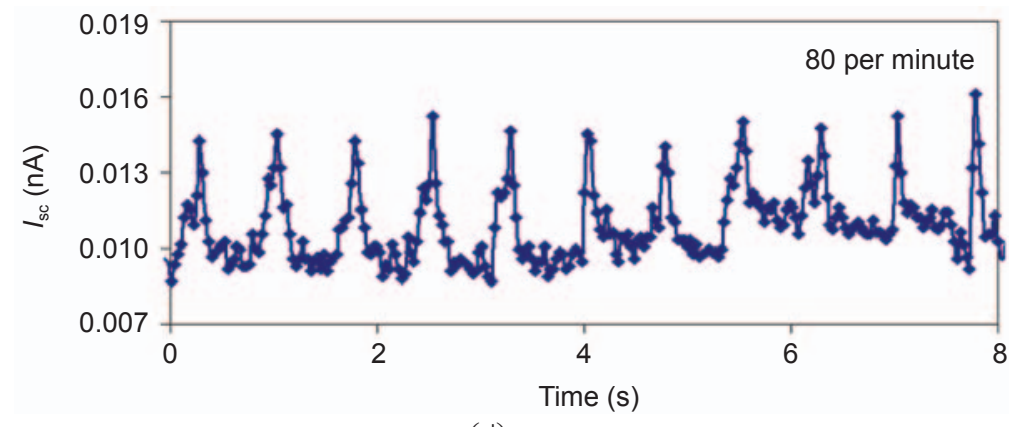

(d)

Figure 7 The design and mechanism of the fiber-based nanogenerator as driven by a low-frequency, external vibration/friction/pulling force. (a) Schematic model of the nanogenerator. (b) Aligned $\mathrm{ZnO}$ nanowire arrays grown on surfaces of a fiber. (c) An SEM image of a pair of entangled fibers. The Au-coated NWs at the top serve as the conductive "tips" that deflect/bend the NWs at the bottom, a piezoelectricsemiconducting couple process generates electric current. (d) Electric current generated by repeatedly sliding the two fibers

in Fig. 7(a). By brushing the metal nanowire arrays across the $\mathrm{ZnO}$ nanowire arrays, the metal wires act like an array of AFM tips that induce the mechanical deformation to the $\mathrm{ZnO}$ nanowires and collect the charges. A unique advantage for $\mathrm{ZnO}$ nanowire arrays is that they can be grown at $80{ }^{\circ} \mathrm{C}$ on substrates of any shape and any materials. In reality, the metal nanowire arrays were made by metal coating of $\mathrm{ZnO}$ nanowire arrays grown on Kevlar fiber (Fig. 7(b)). The metal to be coated is required to form a Schottky contact with $\mathrm{ZnO}$. Entangling the two fibers, one coated with $\mathrm{Au}$ and one without coating, sets the principle of the fiber-based nanogenerator (Fig. 7(c)). A cycled relative sliding between the two fibers produces output current owing to the deflection and bending of the $\mathrm{ZnO}$ nanowires (Fig. 7(d)). This is the fiber-based nanogenerator [22], with potential for harvesting energy from body movement, muscle stretching, light wind, and vibration.

\section{Summary}

In summary, this review presents the various approaches that have been demonstrated for harvesting energy from environment for low-power applications. For nanodevices and nanosystems, the most effective approach is probably the piezoelectric vibration-based generator. By using aligned nanowire arrays, a new nanogenerator has been demonstrated, which has the potential of converting mechanical

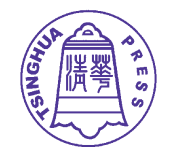


movement energy (such as body movement, muscle stretching, blood pressure), vibration energy (such as acoustic/ultrasonic wave), and hydraulic energy (such as flow of body fluid, blood flow, contraction of blood vessel, dynamic fluid in nature) into electric energy that may be sufficient for self-powering nanodevices and nanosystems. The technology could have important applications in wireless selfpowered nanodevices by harvesting energy from the environment. It also provides a method for indirectly charging of a battery.

\section{Acknowledgements}

This work was supported by DOE BES, NSF, DARPA, and NASA. Thanks to the contributions from my group members: Dr. Jinhui Song, Dr. Xudong Wang, Dr. Yong Qin, Dr. Jin Liu, Dr. Jun Zhou, Dr. Rusen Yang, Yifan Gao, Peng Fei, Dr. Puxian Gao, Dr. Jr-Hau He, Dr. Yifeng Lin, Wenjie Mai, and Prof. Li Shi.

\section{References}

[1] Special issue on Sustainability and Energy, Science 2007, Feb. 9.

[2] Special issue on Harnessing Materials for Energy, MUS Bull. 2008, 33 (4).

[3] Wang, Z. L. Self-powering nanotech. Scientific American 2008, 82-87.

[4] Yu, C.; Hao, Q.; Saha, S.; Shi , L.; Kong, X; Wang, Z. L. Integration of metal oxide nanobelts with microsystems for nerve agent detection. Appl. Phys. Lett. 2005, 86, 063101.

[5] Paradiso, J. A.; Starner, T. Energy scavenging for mobile and wireless electronics. IEEE Pervasive Computing 2005, $14,18-27$.

[6] Leland, E. S.; Baker, J.; Carleton, E.; Reilly, E.; Lai, E.; Otis, B.; Rabaey, J. M.; Wright, P. K.; Sundararajan, V. IEEE Pervasive Computin 2005, 14, 18-28.

[7] Tian, B.; Xiaolin, Z.; Kempa, T. J.; Fang, Y.; Yu, N.; Yu, G.; Huang, J.; Lieber, C. M. Coaxial silicon nanowires as solar cells and nanoelectronic power sources. Nature 2007, 449, 885-890.

[8] Bond, D. R.; Holmes, D. E.; Tender, L. M.; Lovley, D. R. Electrode-reducing microorganisms that harvest energy from marine sediments. Science 2002, 295, 483-485.
[9] Bachand, G. D.; Montemagno, C. D. Constructing organic/ inorganic NEMS devices powered by biomolecular motors. Biomed. Microdevices 2000, 2, 179-185.

[10] Wang, M. D.; Schnitzer, M. J.; Yin, H.; Landick, R.; Gelles, J.; Block, S. M. Force and velocity measured for single molecules of RNA polymerase. Science 1998, 282, 902907.

[11] Kitamura, H.; Tokunaga, M.; Iwane, A. H.; Yanagida, T. A single myosin head moves along an actin filament with regular steps of 5.3 nanometres. Nature 1999, 397, 129134.

[12] Yasuda, R.; Noji, H.; Kinosita, K., Jr.; Yosida, M. F1ATPase is a highly efficient molecular motor that rotates with discrete $120^{\circ}$ steps. Cell 1998, 93, 1117-1124.

[13] Noji, H.; Yasuda, R.; Yoshida, M.; Kinosita, K., Jr. Direct observation of the rotation of F1-ATPase, Nature 1997, 386, 299-302.

[14] Shi, L.; Li, D.; Yu, C.; Jang, W.; Kim, D.; Yao, Z.; Kim, P.; Majumdar, A. Measuring thermal and thermoelectric properties of one-dimensional nanostructures using a microfabricated device. J. Heat Transfer 2003, 125, 881888.

[15] Wang, Z. L.; Song, J. H. Piezoelectric nanogenerators based on zinc oxide nanowire arrays. Science 2006, 312, 242-246.

[16] Song, J. H.; Zhou, J.; Wang, Z. Piezoelectric and semiconducting coupled power generating process of a single $\mathrm{ZnO}$ belt/wire. A technology for harvesting electricity from the environment. Nano Lett. 2006, 6, 1656-1662.

[17] Zhou, J.; Xu, N. S.; Wang, Z. L. Dissolving behavior and stability of $\mathrm{ZnO}$ wires in biofluids: A study on biodegradability and biocompatibility of $\mathrm{ZnO}$ nanostructures. Adv. Mater. 2006, 18, 2432-2435.

[18] Wang, X. D.; Song, J. H.; Liu, J.; Wang, Z .L. Directcurrent nanogenerator driven by ultrasonic waves. Science 2007, 316, 102-105.

[19] Liu, J.; Fei, P.; Zhou, J.; Tummala, R.; Wang, Z. L. Toward high output-power nanogenerator. Appl. Phys. Lett. 2008, in press.

[20] Gao, P. X.; Song, J. H.; Liu, J.; Wang, Z. L. Nanowire nanogenerators on plastic substrates as flexible power source. Adv. Mater. 2007, 19, 67-72.

[21] Qin, Y.; Wang, X. D.; Wang, Z. L. Microfiber-nanowire hybrid structure for energy scavenging. Nature 2008, 451, 809-813. 